ARTICLE

\title{
Dynamics of the 4D genome during in vivo lineage specification and differentiation
}

A. Marieke Oudelaar 1,2,6, Robert A. Beagrie (1) 1,6, Matthew Gosden (1) 1, Sara de Ornellas (1) 1,3, Emily Georgiades (10) 1,2, Jon Kerry², Daniel Hidalgo (1) 4, Joana Carrelha', Arun Shivalingam³, Afaf H. El-Sagheer (10 3,5, Jelena M. Telenius ${ }^{1,2}$, Tom Brown (10 3, Veronica J. Buckle (1) ${ }^{1}$, Merav Socolovsky (1) ${ }^{4}$, Douglas R. Higgs (iD ${ }^{1 凶} \&$ Jim R. Hughes (iD ${ }^{1,2}{ }^{凶}$

Mammalian gene expression patterns are controlled by regulatory elements, which interact within topologically associating domains (TADs). The relationship between activation of regulatory elements, formation of structural chromatin interactions and gene expression during development is unclear. Here, we present Tiled-C, a low-input chromosome conformation capture (3C) technique. We use this approach to study chromatin architecture at high spatial and temporal resolution through in vivo mouse erythroid differentiation. Integrated analysis of chromatin accessibility and single-cell expression data shows that regulatory elements gradually become accessible within pre-existing TADs during early differentiation. This is followed by structural re-organization within the TAD and formation of specific contacts between enhancers and promoters. Our high-resolution data show that these enhancer-promoter interactions are not established prior to gene expression, but formed gradually during differentiation, concomitant with progressive upregulation of gene activity. Together, these results provide new insight into the close, interdependent relationship between chromatin architecture and gene regulation during development.

\footnotetext{
${ }^{1}$ MRC Molecular Haematology Unit, MRC Weatherall Institute of Molecular Medicine, Radcliffe Department of Medicine, University of Oxford, Oxford, UK. ${ }^{2}$ MRC WIMM Centre for Computational Biology, MRC Weatherall Institute of Molecular Medicine, Radcliffe Department of Medicine, University of Oxford, Oxford, UK. ${ }^{3}$ Department of Chemistry, University of Oxford, Oxford, UK. ${ }^{4}$ Department of Molecular, Cell and Cancer Biology, University of Massachusetts Medical School Worcester, Massachusetts, USA. ${ }^{5}$ Department of Science and Mathematics, Faculty of Petroleum and Mining Engineering, Suez University, Suez, Egypt. ${ }^{6}$ These authors contributed equally: A. Marieke Oudelaar, Robert A. Beagrie. ${ }^{凶}$ email: doug.higgs@imm. ox.ac.uk; jim.hughes@imm.ox.ac.uk
} 
E nhancers are non-coding regulatory elements required for precise control of gene expression during mammalian development. The interaction of active enhancer elements with their target genes occurs predominantly within Topologically Associating Domains (TADs), self-interacting regions of chromatin, which are usually $\sim 0.1-1 \mathrm{Mb}$ in size and delimited by convergent binding sites for the zinc-finger protein $\mathrm{CTCF}^{1-3}$. The relationship between enhancer activation, formation of intra-TAD chromatin interactions and gene activation is not completely understood ${ }^{4-6}$. To better understand how genome structure relates to function, it is important to characterize the three-dimensional nuclear architecture of the genome at higher resolution, and to determine how it changes during differentiation and development.

It has been shown that TAD boundaries are generally established early in development and remain relatively stable during differentiation ${ }^{7-10}$. By contrast, interactions within TADs are extensively restructured in differentiating cells, which involves the formation of specific interactions between enhancers and promoters $^{11-15}$. It has been suggested that this reorganization precedes gene activity and that enhancer-promoter interactions are formed prior to gene expression ${ }^{16-18}$. However, due to limitations in temporal and/or spatial resolution in previous studies, it is not known precisely when such interactions are formed during development. The detailed order of events and precise relationship between chromatin architecture and activation of regulatory elements and genes therefore remain unclear. For example, it is possible that enhancers and promoters form limited interactions prior to gene activation due to changes in TAD structure during early differentiation, but that strong upregulation of gene expression is associated with more specific, subtle changes in conformation that will only be detected in data with sufficient resolution and sensitivity.

To better understand the relationship between chromatin architecture and gene expression, it is crucial to characterize chromatin structures at high resolution in pure, primary cell populations representing relevant developmental stages. This has been hampered by the lack of high-resolution Chromosome Conformation Capture (3C) methods that are suitable for the analysis of limited numbers of primary cells. Therefore, we developed Tiled-C, a new 3C-based approach ${ }^{19}$, which can generate high-resolution contact matrices of selected regions of interest from as few as 2000 cells and thereby allows for the analysis of cell populations that have previously been inaccessible.

We have used Tiled-C to study the chromatin architecture of key erythroid genes through sequential stages of in vivo erythroid differentiation in the mouse, including highly purified hematopoietic stem and progenitor cells. In addition, we have generated matched chromatin accessibility and single-cell expression data. We examined six loci, including the a-globin, Slc25a37, Tal1, Cd47, Cpeb4, and Btg2 genes, and focused our analyses on the $\alpha-$ globin genes, because the regulatory elements in this locus are extremely well-characterized. We find that the TAD encompassing the a-globin genes is already present in hematopoietic stem cells. We also find that the first steps in gene activation occur in early committed erythroid progenitors and involve opening of the a-globin enhancers, which become accessible prior to both chromatin reorganization and activation of $a$-globin RNA expression. Subsequent chromatin reorganization involves the appearance of smaller self-interacting domains (sub-TADs) within the larger $\mathrm{TAD}$, in which specific interactions between enhancers and promoters are formed. In contrast to the current literature ${ }^{16-18}$, we find that these enhancer-promoter interactions do not precede upregulation of gene activity, but are formed gradually and concomitantly with progressive activation of $\alpha$ globin expression. Importantly, we find a similar order of events at other erythroid gene loci. Therefore, our data demonstrate that -at this improved level of resolution-chromatin architecture and gene activation are more tightly linked than previously appreciated. Together, these findings provide new insights into the mechanisms contributing to the establishment of tissuespecific chromatin structures during development.

\section{Results}

Tiled-C generates low-input and high-resolution $3 \mathrm{C}$ data. We developed Tiled-C, a 3C technique, which generates deep, highresolution contact matrices of genomic regions of interest. TiledC maximizes library complexity by employing a single-tube protocol for 3C library preparation, which minimizes losses during the procedure ${ }^{20}$. This is combined with an enrichment approach derived from the efficient Capture- $\mathrm{C}$ technology, which allows for up to a million-fold enrichment of interactions of interest $^{21,22}$. While Capture-C targets individual restriction fragments as viewpoints, Tiled-C uses a panel of capture oligonucleotides tiled across all restriction fragments of specified genomic regions to efficiently enrich for contacts within this region. This allows for deep, targeted sequencing of chromatin interactions within regions of interest and thus for the generation of high-resolution contact matrices at unprecedented depth, across multiplexed samples and genomic regions. Tiled- $\mathrm{C}$ therefore combines the ability of all vs all methods such as $\mathrm{Hi}-\mathrm{C}^{23}$ to map large-scale chromatin structures including TADs, and the ability of one vs all methods such as $4 C^{24,25}$ and Capture- $C^{21,22}$ to robustly identify enhancer-promoter interactions within TADs in detail (Supplementary Figs. 1 and 2). To validate the Tiled-C approach, we compared Tiled-C data to the deepest currently available in situ Hi-C datasets (mouse ES cells ${ }^{9}$; Fig. 1a). Tiled-C data at this region was $\sim 28$-fold higher in depth and required $\sim 19$-fold less sequencing (Supplementary Table 1). To further demonstrate the capabilities of Tiled-C, we performed experiments in which we multiplexed across several key gene loci in both primary mouse erythroid cells and ES cells, to characterize their tissue-specific configurations in depth (Fig. 1b, c).

Current methods, such as Targeted Chromatin Capture $(\mathrm{T} 2 \mathrm{C})^{26}$, Capture Hi-C ${ }^{27}$, and HYbrid Capture Hi-C $\left(\mathrm{Hi}_{-} \mathrm{C}^{2}\right)^{28}$ also use an oligonucleotide capture procedure to enrich $3 \mathrm{C}$ or $\mathrm{Hi}$ $\mathrm{C}$ libraries for regions of interest (Supplementary Table 2). These methods require millions of cells per sample. Tiled-C is specifically designed to maximize the efficiency of the experimental procedure and its increased sensitivity allows for the generation of reproducible, high-resolution contact matrices from as few as 2000 cells (Fig. 2, Supplementary Figs. 3 and 4, Supplementary Table 3), thereby enabling the analysis of previously intractable primary cell types. This is critical for the investigation of $4 \mathrm{D}$ (3D structure through developmental time) genome organization, as cell numbers become extremely limiting at early stages of development.

We used Tiled-C to study changes in chromatin structure associated with gene activation in primary cells during in vivo mouse erythropoiesis. We initially focused on the a-globin cluster because the a-globin genes and their regulatory elements are extremely well-characterized. The mouse a-globin cluster comprises the duplicated adult a-globin genes $\mathrm{Hba-1}$ and $\mathrm{Hba-2}$, as well as the embryonic gene $\mathrm{Hba-x}$, and two genes of unknown function $H b q-1$ and $H b q$-2. These genes are located in a TAD, which also contains five additional genes upstream of the $\alpha$ globin cluster: Nprl3, Mpg, Rhbdf1, Snrnp25, and Il9r. The aglobin genes are regulated by five erythroid-specific enhancer elements (R1-R4 and Rm), which classify as a super-enhancer ${ }^{29}$. In terminally differentiating erythroblasts these enhancers interact with the gene promoters in a sub-TAD flanked by multiple 


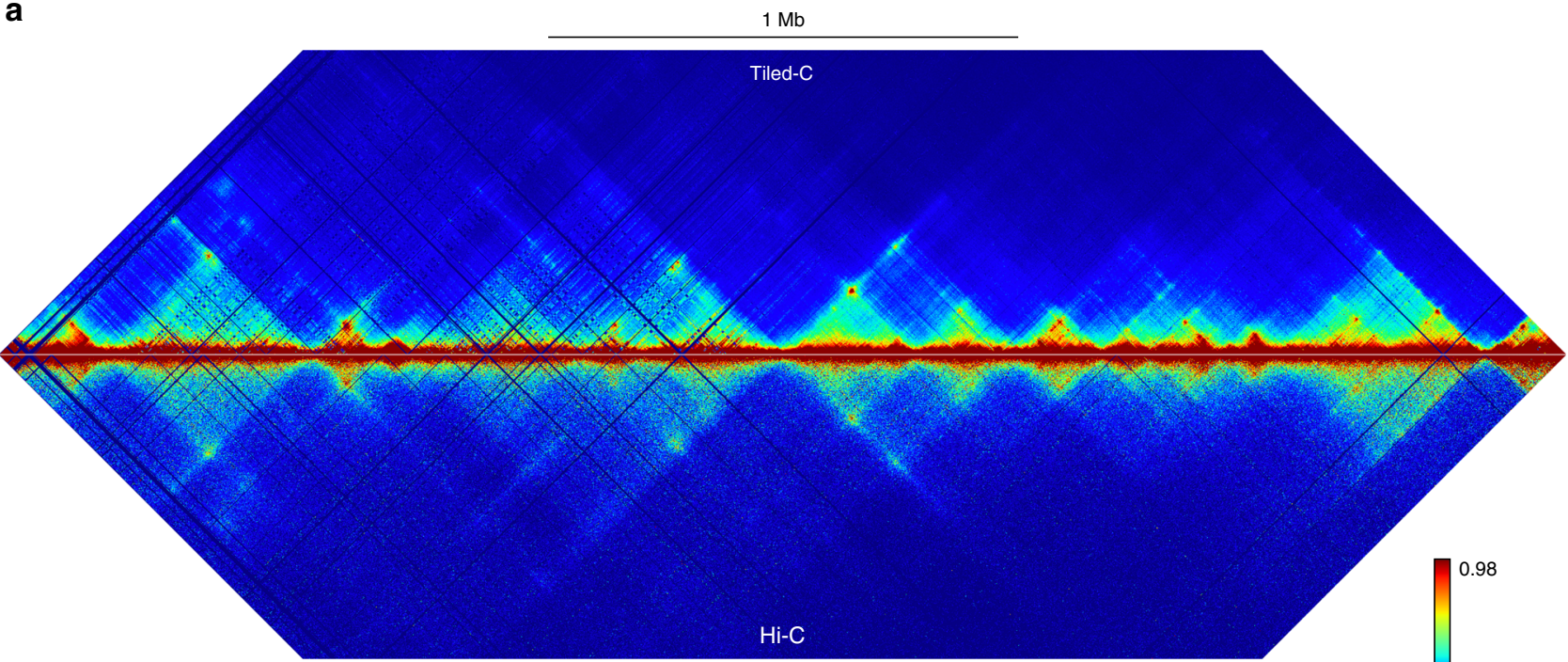

b
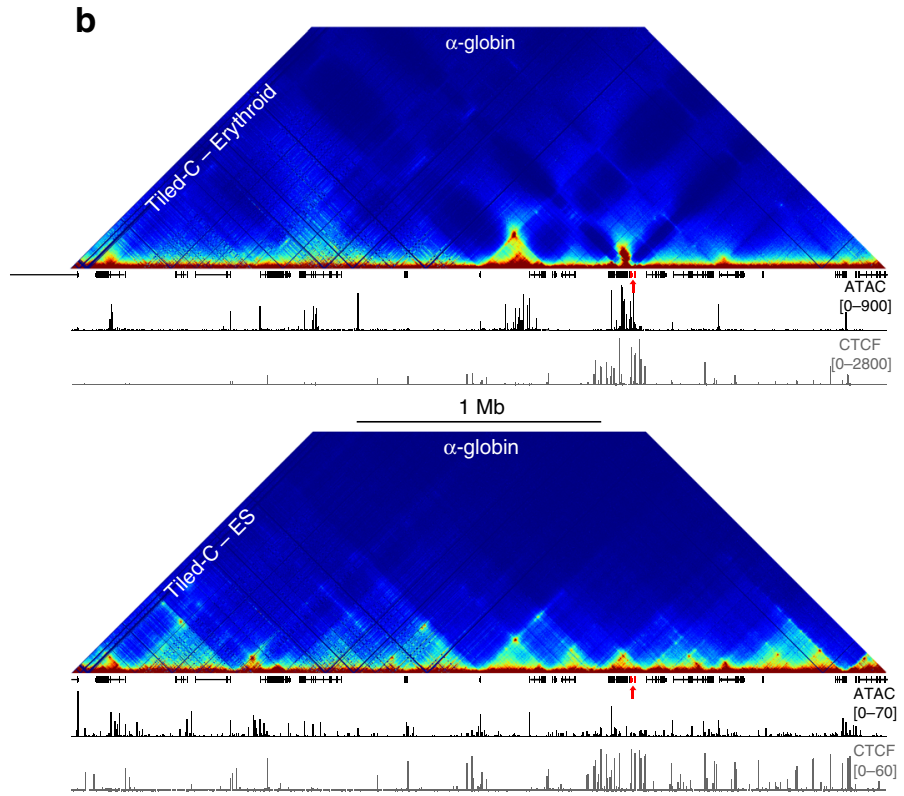
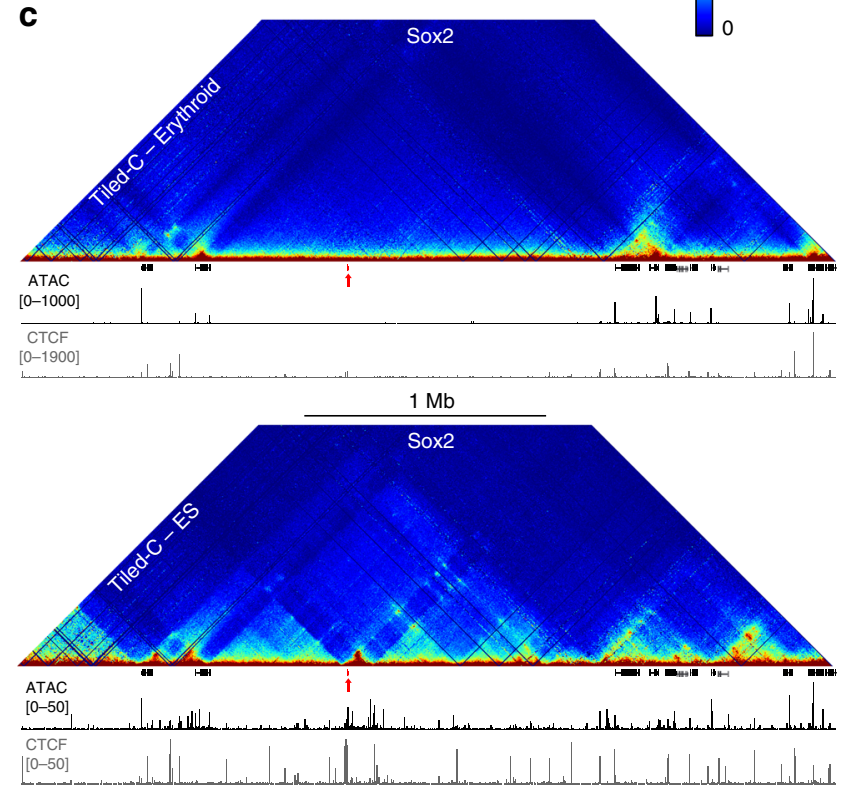

Fig. 1 Tiled-C generates deep all vs all $3 \mathrm{C}$ data at regions of interest. a Comparison of Tiled-C and $\mathrm{Hi}-\mathrm{C}$ contact matrices at $2 \mathrm{~kb}$ resolution in mouse $\mathrm{ES}$ cells. Contact frequencies represent normalized, unique interactions in three and four replicates for Tiled-C and Hi-C data, respectively. Coordinates (mm9): chr11:29,902,000-33,228,000. b Tiled-C contact matrices of $\sim 3.3 \mathrm{Mb}$ spanning the mouse $\alpha$-globin locus in primary mature erythroid cells (top) and ES cells (bottom) at $2 \mathrm{~kb}$ resolution. Contact frequencies represent normalized, unique interactions in three replicates. Gene annotation ( $\alpha$-globin genes highlighted in red), open chromatin (ATAC), and CTCF occupancy are shown below the matrices. Coordinates (mm9): chr11:29,902,000-33,228,000. c Tiled-C contact matrices of $\sim 3.4 \mathrm{Mb}$ spanning the mouse Sox2 locus in primary mature erythroid cells (top) and ES cells (bottom) at $5 \mathrm{~kb}$ resolution. Contact frequencies represent normalized, unique interactions in four replicates. Gene annotation (Sox2 gene highlighted in red), open chromatin (ATAC), and CTCF occupancy are shown below the matrices. Coordinates (mm9): chr3:33,200,000-36,565,000.

CTCF-binding elements, which are predominantly in a convergent orientation ${ }^{30-32}$ (Supplementary Fig. 1).

Isolation of primary erythroid cell populations. Using fluorescence-activated cell sorting (FACS), we isolated cells at sequential stages of erythroid differentiation directly from mouse fetal livers (Fig. 3a, b). This allowed us to analyze highly purified primary cells through in vivo erythropoiesis. The S0-low cell population consists of early progenitors, predominantly BurstForming Unit-Erythroid (BFU-E) cells. S0-medium consists primarily of early Colony-Forming Unit-Erythroid (CFU-E) cells, while S1 and S2 contain the last CFU-E cell division before terminal differentiation ${ }^{33,34}$. S3 through to S5 consist of terminally differentiating erythroblasts in progressively more mature states. Because erythroid cells enucleate in the final stages of differentiation, we have focused our analyses on stages S0 through to S3. In vitro, differentiation from S0 cells to S1 cells takes about $10 \mathrm{~h}$, and differentiation to S3 cells takes an additional $10 \mathrm{~h}$.

Gene expression through erythroid differentiation. We used Cellular Indexing of Transcriptomes and Epitopes by Sequencing (CITE-seq $)^{35}$, a variant of single-cell RNA-seq, to characterize gene expression in the isolated cell populations. We generated a dataset that covers the full course of in vivo erythroid differentiation through to terminal differentiation in the mouse (Supplementary Fig. 5), which shows that a-globin is expressed at basal levels in the S0 populations. Expression of a-globin dramatically increases during the S2 stage and plateaus at S3; 

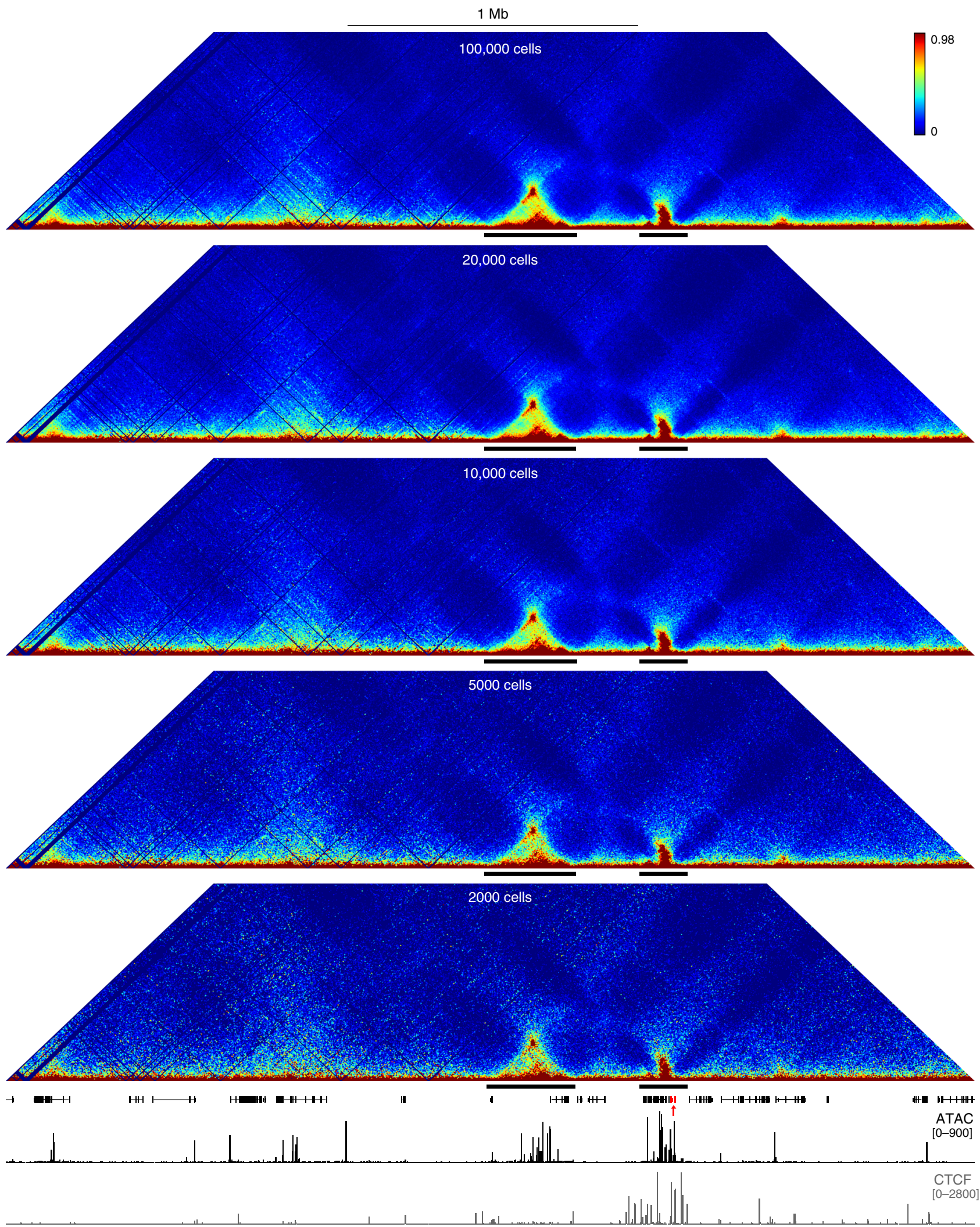

Fig. 2 Tiled-C generates high-resolution contact matrices from small numbers of cells. Tiled-C contact matrices of $\sim 3.3 \mathrm{Mb}$ spanning the mouse $\alpha$-globin locus at $5 \mathrm{~kb}$ resolution, generated from small aliquots of primary mature erythroid cells. Contact frequencies represent normalized, unique interactions in three replicates. TADs are indicated below each matrix with a black bar. Gene annotation ( $\alpha$-globin genes highlighted in red), open chromatin (ATAC), and CTCF occupancy are shown below the matrices. Coordinates (mm9): chr11:29,900,000-33,230,000. 

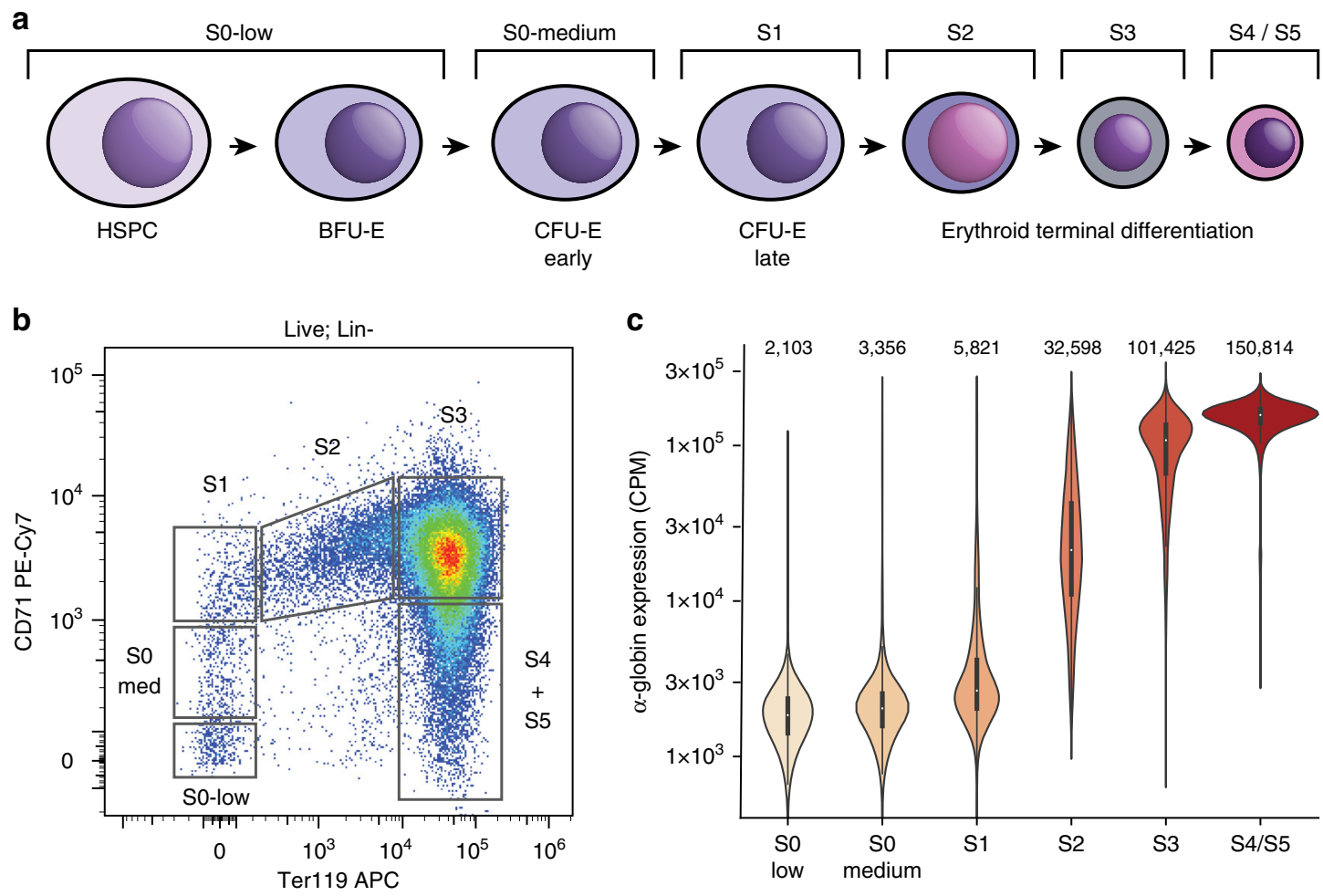

C
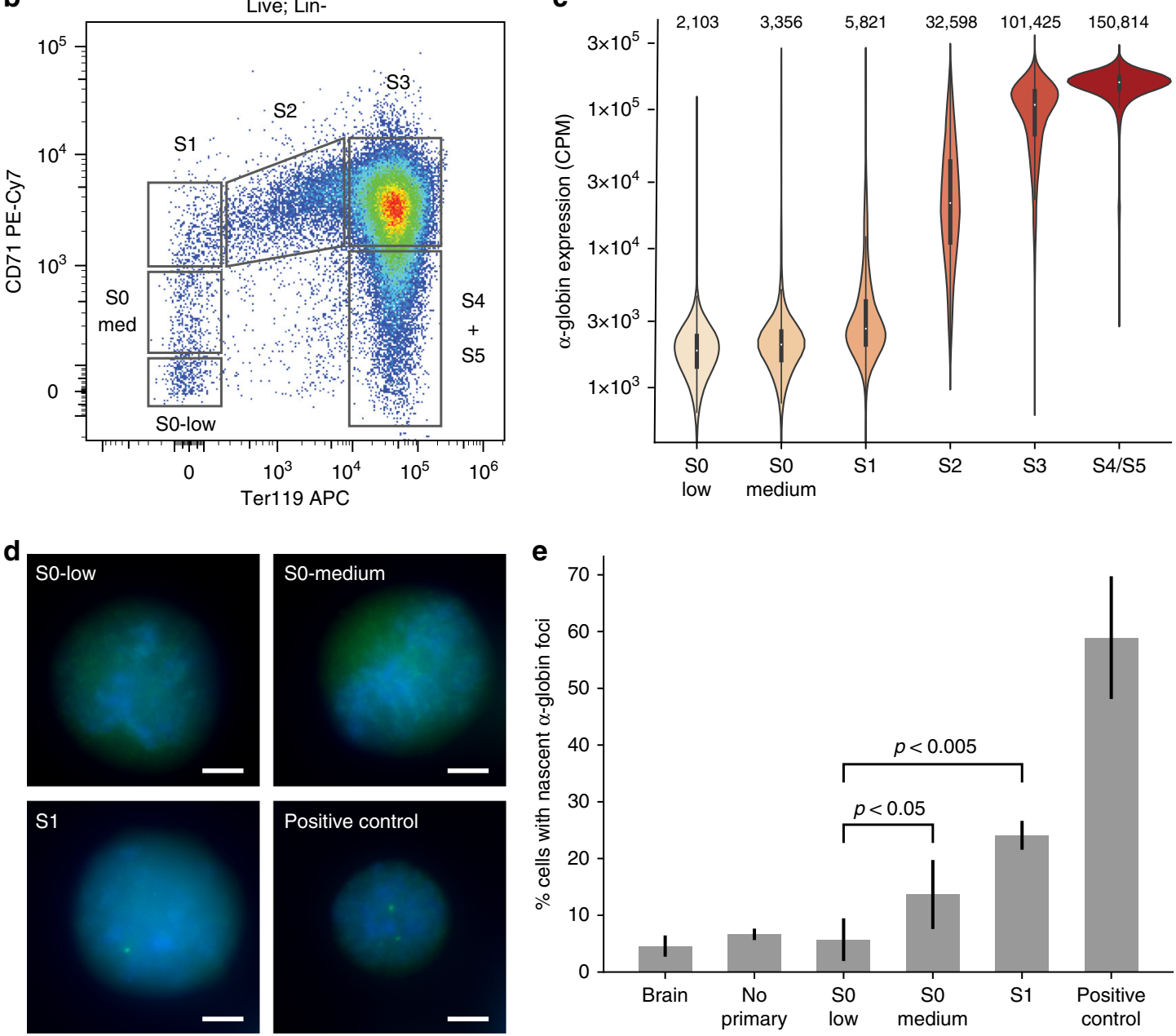

Fig. 3 Expression of $\boldsymbol{\alpha}$-globin is gradually upregulated during in vivo erythroid differentiation. a Scheme of erythroid differentiation showing the various populations analyzed. $\mathbf{b}$ Example FACS plot showing the gating strategy used to isolate erythroid progenitors from mouse fetal liver. $\mathbf{c}$ Expression (in counts per million) of $\alpha$-globin transcripts in each population as determined by single-cell RNA-seq. Mean expression for each population is given at the top of each bar. $\mathbf{d}$ Representative RNA-FISH images showing detection of nascent $\alpha$-globin transcripts in sorted early erythroid progenitors. Scale bar is $3 \mu \mathrm{M}$ for each image. e RNA-FISH quantification, showing the mean \pm s.d. of $n=3$ independent experiments (except for brain and "no primary" negative controls, which have $n=2$ ). $P$-values were calculated by two-tailed paired $t$-tests (SO-low vs S0-medium $P=0.041$, S0-low vs S1 $P=0.002$ ). Source data are provided as a Source Data file.

however, the earliest cells showing elevated expression of a-globin are found in the S1 stage (Fig. 3c). To validate that erythroidspecific a-globin upregulation begins at S1, we performed RNAFISH to detect nascent transcription in FACS-sorted primary cells (Fig. 3d). We detect a small increase in nascent transcription from S0-low to S0-medium cells and confirm a robust increase in expression from S0 to S1 cells $(P<0.005$ by paired $t$-test; Fig. $3 \mathrm{e})$.

Chromatin architecture through erythroid differentiation. We used the Assay for Transposase-Accessible Chromatin using sequencing (ATAC-seq) ${ }^{36}$ to profile chromatin accessibility in these stages. Interestingly, we find that both enhancer and promoter elements are accessible prior to the onset of erythroidspecific gene expression, and that the degree of accessibility gradually increases, concomitant with upregulation of a-globin expression (Fig. 4, Supplementary Fig. 6). Tiled-C analysis shows that a TAD structure encompassing the a-globin locus is present at the earliest stage (S0-low), prior to the formation of weak enhancer-promoter interactions in the S0-medium stage (Fig. 4, Supplementary Figs. 7 and 8). These enhancer-promoter interactions strengthen in the subsequent S1 and S2 stages, accompanied by increases in a-globin expression and accessibility. In the S3 stage, where chromatin accessibility and expression reach their maximum levels, we observe a further increase in enhancer- 


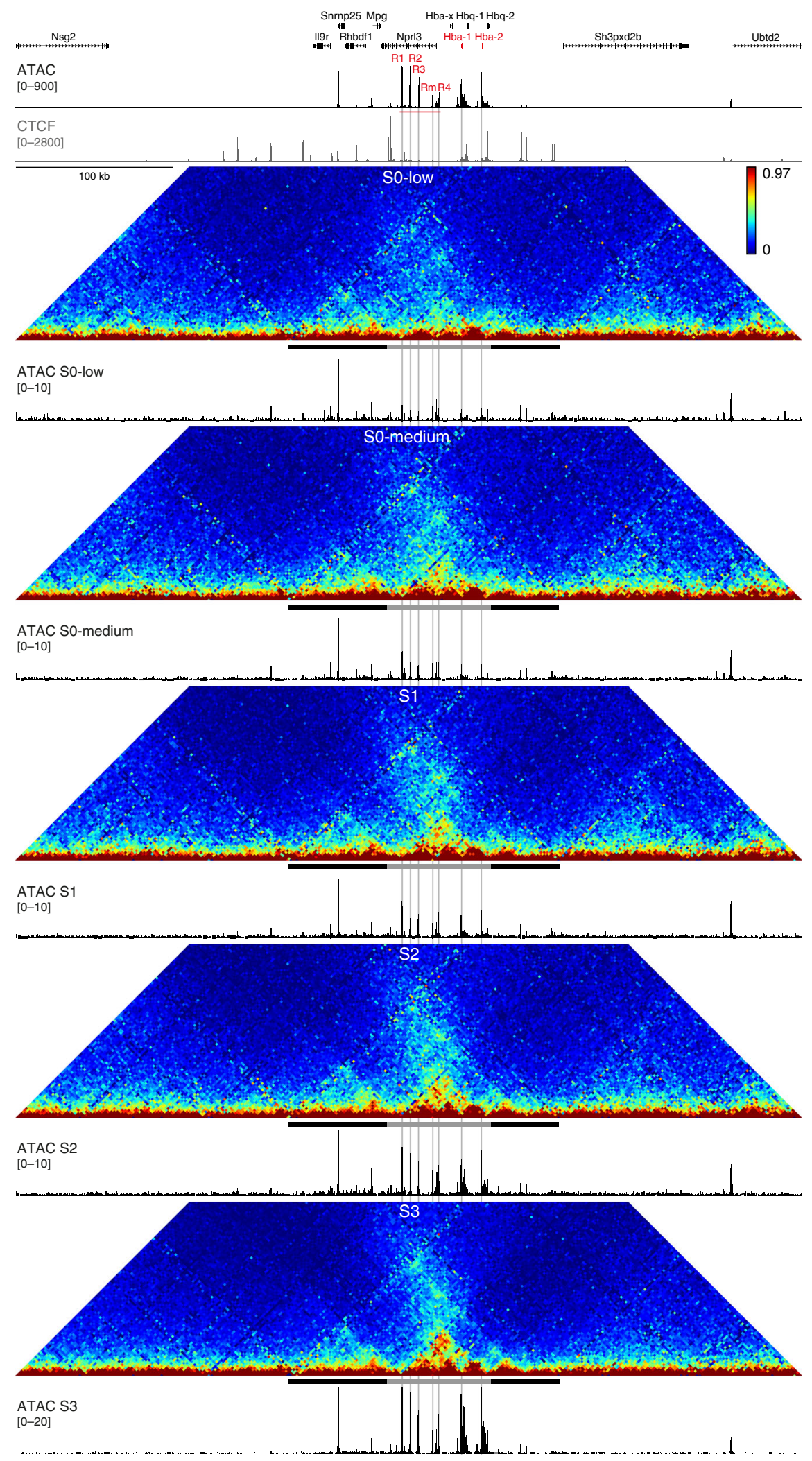

promoter interactions in a sub-domain within the TAD, similar to the structure observed in primary erythroblasts derived from mature spleen tissue (Fig. 1, Supplementary Figs. 1 and 2). The smaller sub-TAD, which gradually forms within the pre-existing TAD, is delimited by convergent CTCF-binding elements that flank the a-globin enhancers and genes (Fig. 4, Supplementary Fig. 1). We have previously shown that these CTCF-binding elements are functionally important to restrict the interactions of the a-globin enhancers and prevent other genes within the TAD, but outside the sub-TAD, from being upregulated ${ }^{31,37}$. This suggests that this smaller erythroid-specific domain is likely formed by similar CTCF-dependent mechanisms as TADs, although it is smaller in size $(\sim 70 \mathrm{~kb})$ and has very high internal interaction frequencies compared to typical TADs. 
Fig. 4 Upregulation of $\alpha$-globin expression correlates with increased chromatin accessibility and enhancer-promoter interactions. Tiled- $C$ contact matrices of $500 \mathrm{~kb}$ spanning the mouse $\alpha$-globin locus in sequential stages of in vivo erythroid differentiation at $2 \mathrm{~kb}$ resolution. Contact frequencies represent normalized, unique interactions in two replicates. The black and gray bar below each matrix represent the pre-existing TAD (chr11:32,080,000$32,245,000$ ) and erythroid-specific sub-TAD (chr11:32,136,000-32,202,000), respectively. Matched open chromatin (ATAC) profiles are shown underneath the matrices and represent normalized data from 3 SO-low, S0-medium, and S1 replicates and 2 S2 and S3 replicates. The ATAC profiles are shown at different scales to highlight changes in accessibility in early stages of differentiation. Gene annotation ( $\alpha$-globin genes highlighted in red), open chromatin (ATAC; $\alpha$-globin enhancers highlighted in red), and CTCF occupancy in mature mouse erythroblast cells are shown at the top. Coordinates (mm9): chr11:31,900,000-32,400,000.
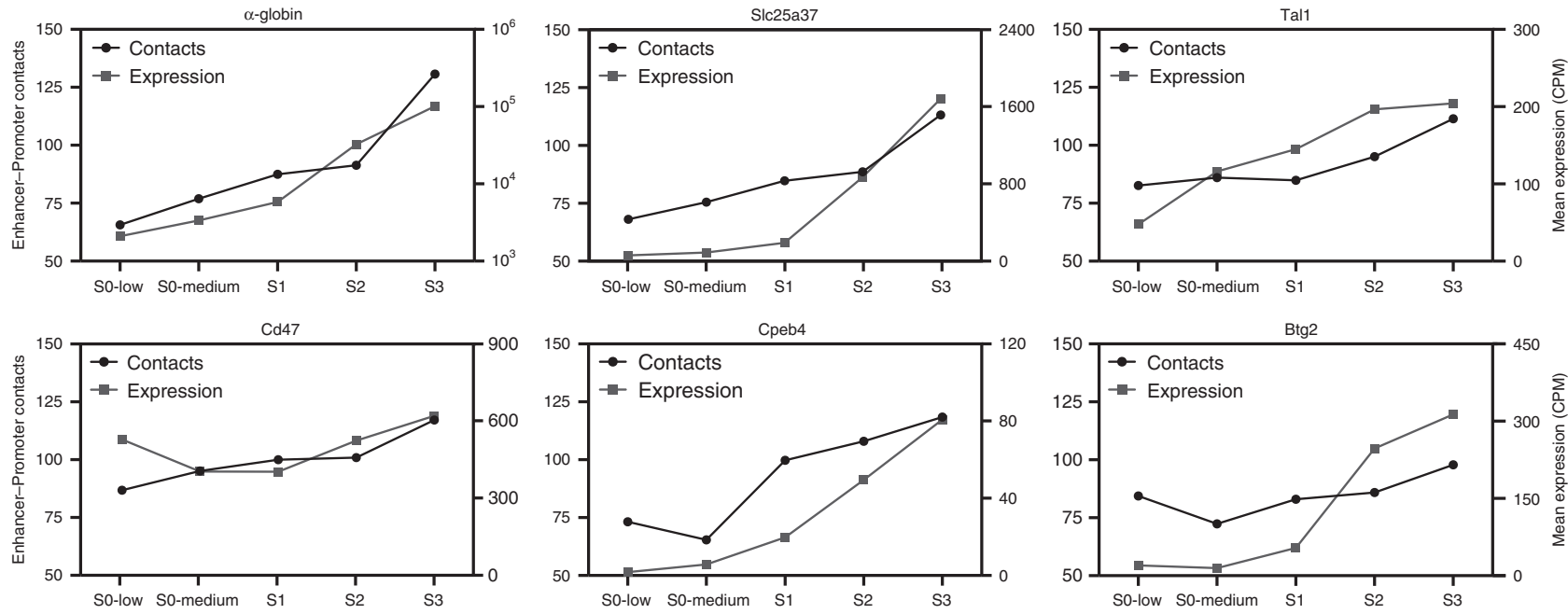

Fig. 5 Enhancer-promoter interactions are formed progressively during erythroid differentiation and correlate with upregulation of gene expression.

Quantification of enhancer-promoter interactions and gene expression during in vivo erythroid differentiation at six erythroid gene loci. Contact frequencies (black circles; left $Y$-axis) represent unique interactions normalized for the total number of contacts in the matrix. Expression counts (gray squares; right $Y$ axis) represent mean expression in counts per million (CPM) for each population as determined by single-cell RNA-seq. Expression of $\alpha$-globin, SIc25a37 and Tal1, is upregulated early in differentiation, concomitant with increased enhancer-promoter interactions. Cd47 is expressed in hematopoietic stem cells and further upregulated later in erythroid differentiation when enhancer-promoter interactions are strengthened. Cpeb4 and Btg2 become robustly expressed in the S1 stage and are further upregulated as enhancer-promoter interactions increase later in differentiation.

Since both accessibility and the encompassing TAD structure are present prior to erythroid-specific a-globin expression, we purified early hematopoietic progenitor populations to investigate when in differentiation these features are established. Interestingly, we find that the pre-existing TAD containing the $\alpha$-globin locus is already present in hematopoietic stem cells, despite four out of five enhancers and both promoters being inaccessible at this stage (Supplementary Fig. 9).

To examine whether a similar order of events operates at other gene loci, we examined the chromatin architecture of five additional erythroid gene loci through erythropoiesis: Slc25a37, Tal1, Cd47, Cpeb4, and Btg2. In each of these loci, we find that regulatory elements are accessible prior to gene activation and gradually increase in accessibility as gene expression is upregulated (Supplementary Figs. 10-12). We also find that these elements interact at basal levels in a pre-existing TAD structure prior to gene expression. However, specific interactions between the regulatory elements progressively increase during differentiation as erythroid-specific gene activity increases (Fig. 5). These results confirm that specific regulatory interactions do not precede gene activation, but are formed gradually and concomitant with upregulation of gene expression.

\section{Discussion}

The mouse $\alpha$-globin cluster has a long history as model locus for studying gene regulation during differentiation and development $^{38}$. Previous analysis of transcription factor binding at the $\alpha$ - globin locus has shown that lineage commitment and differentiation are driven by sequential appearance of key transcription factors ${ }^{39}$, consistent with the gradual increase in chromatin accessibility at the regulatory elements we describe here. In addition, previous 3C studies of both the $\alpha$ - and $\beta$-globin loci in erythroid cell lines have demonstrated that interactions between enhancers and promoters are tissue-specific ${ }^{40,41}$. More recently, the tissue-specific conformation of the a-globin locus has also been described based on super-resolution imaging of two stages of ex vivo erythroid differentiation ${ }^{42}$.

A limitation of the current body of work on chromatin organization during differentiation and development-both at the globin clusters and other gene loci-is that experiments have been performed at low spatial and temporal resolution and predominantly in vitro. Previous studies have therefore not been able to identify at what point in differentiation tissue-specific interactions between enhancers and promoters are established, nor how the formation of these interactions relates to changes in gene expression. Progress in this area has been limited by a lack of techniques capable of generating high-resolution interaction data from the small numbers of cells available in developmentally relevant primary cell populations.

To overcome this hurdle, we have developed Tiled-C, a new 3C-based approach, which can generate high-resolution chromatin interaction data from as few as 2000 cells and thus enables analysis of cell populations that have previously been inaccessible. We have used Tiled-C, in combination with ATAC and single-cell RNA-seq, to study the dynamic chromatin architecture and 

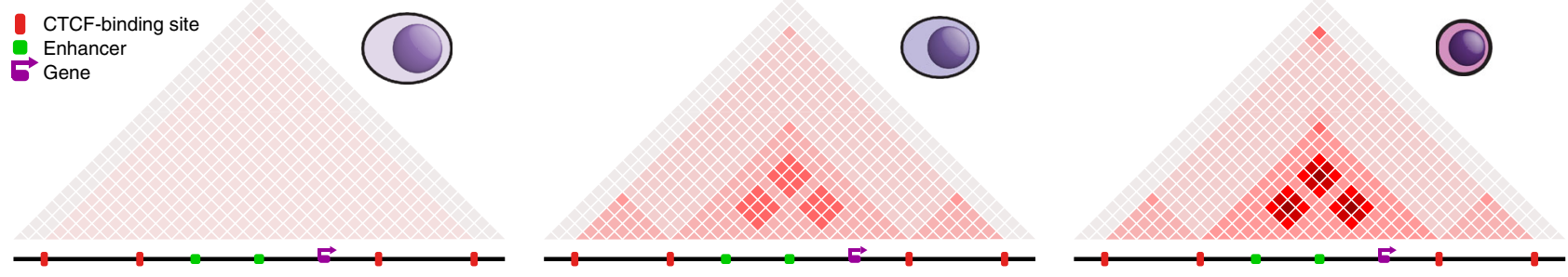

Fig. 6 Graphical Summary. Based on our findings, we propose a model in which TADs are established very early in differentiation. During lineage commitment, tissue-specific open chromatin sites are established within these domains. This is followed by the formation of smaller sub-domains within TADs, in which enhancers and promoters form interactions. Through differentiation, accessibility and specific interactions between enhancers and promoters are gradually increased, concomitant with upregulation of gene expression.

expression of the $\alpha$-globin cluster through in vivo erythropoiesis. Our data show that the boundaries of the TAD containing the $\alpha$ globin cluster are already established in hematopoietic stem cells and maintained during further differentiation. This is consistent with previous reports, which have shown that TADs are relatively stable during differentiation and development ${ }^{7-10}$. In contrast to the current literature ${ }^{16-18}$, however, the high resolution of our data has enabled us to show that the formation of smaller domains within TADs and the subtle structural changes that strengthen specific enhancer-promoter interactions both occur gradually during erythroid differentiation, concomitant with progressive upregulation of gene activity (Fig. 6). Interestingly, initial chromatin accessibility is detectable at the regulatory elements of the a-globin locus prior to conformational change and activation of gene expression, although accessibility also increases gradually during differentiation. In addition to the a-globin cluster, we demonstrate the same order of events across five other erythroid gene loci.

It is likely that the formation of specific sub-domains within TADs early in differentiation facilitates interactions between enhancers and promoters to prime loci for gene activation. It has recently been shown for the globin loci that gene activation is associated with the formation of higher-order hub-like structures, in which multiple enhancers and promoters form simultaneous, specific interactions ${ }^{30,43}$. Our data suggest that these structures may only be formed in the final stages of differentiation, when chromatin accessibility and interactions between enhancers and promoters are strongest, and may be important to achieve maximal gene expression. This is further supported by recent live imaging experiments in Drosophila, in which gene activation only occurred upon the formation of tight associations between enhancers and a gene promoter, and not after induced enhancerpromoter proximity resulting from interactions between insulator elements $^{44}$.

This model implies that there are multiple processes contributing to the formation of specific chromatin structures associated with gene activation. A pre-existing TAD encompassing the a-globin locus is formed early in differentiation. This occurs prior to activation of the regulatory elements of a-globin, though other genes within the domain are active at this early stage. The formation of the TAD is likely driven by tissue-invariant loop extrusion mediated by cohesin and constitutive CTCF-binding elements $^{28,45}$. During differentiation, chromatin accessibility increases, and a smaller sub-domain is formed within this TAD. We have previously shown that deletion of the CTCF-binding sites at the base of this sub-domain causes it to expand and leads to aberrant expression of the neighboring genes ${ }^{31,37}$. This indicates that the boundaries of the sub-TAD are dependent on these CTCF-binding sites and implies that its formation is mediated by loop extrusion. Since the CTCF-binding sites are constitutively occupied, erythroid-specific domain formation is likely driven by increased rate or processivity of loop extrusion in this region during differentiation. As we have previously observed erythroid-specific accumulation of cohesin at the a-globin enhancers $^{31}$, it is possible that this is mediated by increased cohesin recruitment or stalling at the activated regulatory elements. This is further supported by studies showing that cohesin colocalizes with transcription factors across the genome ${ }^{46,47}$.

The initial appearance of chromatin accessibility at the regulatory elements of a-globin occurs early in differentiation and significantly precedes the onset of specific enhancer-promoter interactions. This suggests that chromatin opening can occur independently of larger scale chromatin reorganization, yet further increases in accessibility do occur alongside the establishment and progressive strengthening of enhancer-promoter interactions, suggesting only a partial decoupling. Our observation that the TAD at the a-globin locus is present prior to accessibility of the regulatory elements indicates that activation of regulatory elements is not required for the establishment of the TAD, which is consistent with our previous work showing that deletion of the a-globin enhancers has no impact on the formation of the $\alpha$-globin TAD. In contrast, the $\alpha$-globin enhancer deletions do affect specific interactions between the a-globin promoters and the remaining enhancers ${ }^{29,42}$, reinforcing that regulatory elements play a role in the formation of tissue-specific chromatin structures, possibly mediated by interactions between the multi-protein complexes bound at these elements.

In conclusion, our dissection of the chromatin architecture of a well-understood gene locus through in vivo erythroid differentiation demonstrates that chromatin architecture and gene activation are tightly linked during development and provides new insights into the distinct mechanisms contributing to the establishment of tissue-specific chromatin structures. Importantly, Tiled-C provides an approach that enables such detailed analysis in cell types that were previously intractable.

\section{Methods}

Cells. Mature primary Ter $119+$ erythroblasts were obtained from spleens of female C57BL/6 mice treated with phenylhydrazine as previously described ${ }^{22}$. Mouse ES cells (E14 and A9 lines) were cultured and harvested as previously described $^{22}$.

Primary erythroid progenitor cells were isolated from fetal livers, which were freshly isolated at e12.5-e13.5 from C57BL/6 mouse embryos. 5-15 livers were pooled together for each experimental replicate, mechanically dissociated in staining buffer (PBS, $0.2 \%$ BSA, $5 \mathrm{mM}$ Glucose) and strained through a $30-\mu \mathrm{m}$ 
strainer. Cells were immunostained at $4{ }^{\circ} \mathrm{C}$ in the presence of rabbit IgG $(200 \mu \mathrm{g} /$ $\mathrm{ml}$, Jackson Laboratories 015-000-003) to block Fc receptors. To enrich for early erythroid progenitors, cells were first stained with $5 \mu \mathrm{g} / \mathrm{ml}$ biotin-conjugated antiTer119 (BD 553672) for $30 \mathrm{~min}$, before magnetic depletion using streptavidin nanobeads (BioLegend Mojosort 480016) following the manufacturer's instructions. Cells were then incubated with $0.5 \mu \mathrm{g} / \mathrm{ml}$ APC-conjugated streptavidin (BD 553672), $0.33 \mu \mathrm{g} / \mathrm{ml} \mathrm{PE-Cy7-conjugated} \mathrm{anti-CD71} \mathrm{(BioLegend}$ 113811 ) and a panel of 5 FITC-conjugated lineage antibodies (anti-CD41, antiCD45R, anti-CD3e, anti-CD11b and anti-Ly-6G/6C, all at $1 \mu \mathrm{g} / \mathrm{ml}$; BD 553848, 553087, 553061, 557396, and 553126, respectively) for $45 \mathrm{~min}$. Cells were then resuspended in FACS running buffer (staining buffer plus $2 \mathrm{mM}$ EDTA). $0.66 \mu \mathrm{g} /$ $\mathrm{ml}$ Hoechst was added immediately prior to sorting in order to distinguish live cells. Cells were sorted into Eppendorf tubes containing $500 \mu \mathrm{l}$ RPMI supplemented with $10 \%$ FCS using a BD FACSAria ${ }^{\text {tw }}$ Fusion machine with a $100 \mu \mathrm{M}$ nozzle size.

Hematopoietic stem and progenitor cells (HSPCs), lymphoid-primed multipotential progenitors (LMPPs) and multipotent progenitors (MPPs) were stained and FACS sorted from the bone marrow of 12-week-old female C57BL/6 mice as previously described ${ }^{48}$.

All protocols were approved through the Oxford University Local Ethical Review process and all experimental procedures were performed in accordance with European Union Directive 2010/63/EU and/or the UK Animals (Scientific Procedures) Act, 1986.

Replicates. The presented Tiled-C data derived from mature splenic erythroblasts and ES cells represent biological triplicates produced from separate mice or culture flasks, respectively. The presented Tiled-C data derived from hematopoietic and erythroid progenitor populations represent biological duplicates, with the exception of the S1 stage, for which we used a single biological replicate to generate technical duplicates. The presented ATAC data derived from hematopoietic and erythroid progenitor populations represent biological triplicates for the S0-low, S0medium, and S1 populations, biological duplicates for the S2 and S3 populations, and single replicates for the hematopoietic progenitor populations. The presented RNA-FISH data represent biological triplicates except for the brain and no primary-antibody-negative control, which represent biological duplicates.

Tiled-C-rationale. Tiled-C is a hybrid of the all vs all $3 \mathrm{C}$ methods, such as Hi$\mathrm{C}^{23}$, and the one vs all methods, such as $4 \mathrm{C}^{24,25}$ and Capture- $\mathrm{C}^{21,22}$. Tiled-C generates all vs all contact matrices of specified genomic regions and thus combines an unbiased all vs all view with the ability to target regions of interest, without the need to sequence chromatin interactions genome-wide. Tiled-C has similarities to $5 \mathrm{C}^{49}$ and Targeted Chromatin Capture (T2C) ${ }^{26}$. However, Tiled-C allows for reliable PCR duplicate filtering based on random sonication ends and uses more efficient capture enrichment, and is therefore able to generate data at higher resolution and depth. Tiled-C also has similarities to Capture $\mathrm{Hi}-\mathrm{C}^{27}$ and HYbrid Capture $\mathrm{Hi}-\mathrm{C}\left(\mathrm{Hi}-\mathrm{C}^{2}\right)^{28}$, which use oligonucleotide capture to enrich Hi-C libraries for regions of interest (Supplementary Table 2). The main differences are that these methods enrich a biotinylated Hi-C library, while Tiled-C enriches a $3 \mathrm{C}$ library generated with an optimized procedure to retain maximal library complexity, which is critical for the analysis of small cell numbers. In addition, Tiled-C uses an efficient capture oligonucleotide design targeted directly to the ends of all restriction fragments present in the region of interest and an efficient capture enrichment procedure enabling up to a million-fold enrichment of restriction fragments of interest. The combination of high library complexity and efficient enrichment in Tiled-C enables high-resolution data generation at great depth and makes the method suitable for the analysis of small cell numbers. Moreover, enriching for targeted regions of interest substantially decreases sequencing costs. We should note, however, that the synthesis of large amounts of capture oligonucleotides can also be expensive. We therefore believe that Tiled-C is particularly useful for researchers who are interested in studying genomic regions of interest in multiple replicates and conditions and/or in primary cells with limited availability.

Tiled-C-oligonucleotide design. Tiled-C uses a panel of $70 \mathrm{bp}$ oligonucleotides to enrich for regions of interest. The oligonucleotide sequences are designed complementary to both ends of each individual restriction fragment present in the region of interest. We use stringent BLAT-based filtering to ensure that the panel of oligonucleotides does not contain repetitive sequences that would decrease specific enrichment of the region of interest. To help users design capture oligonucleotides, we have developed a user-friendly python package (https://oligo.readthedocs.io/en/ latest/). An overview of the oligonucleotide designs used in our study is shown in Supplementary Table 4 .

Tiled-C-oligonucleotide synthesis. The enrichment step in the Tiled-C protocol can be performed with single-stranded or double-stranded biotinylated capture oligonucleotides (Supplementary Fig. 4, Supplementary Table 5).

We produced panels of single-stranded oligonucleotides on an in-house Combimatrix CustomArray B3 DNA synthesiser (B3Synth_v25.1 software) using CustomArray $12 \mathrm{~K}$ Blank Slides (CustomArray Inc., PN: 2000100- Oligo pool Application). All the probe sequences were designed to be 70 bases in length and were placed at random positions on the microarray for synthesis using Layout
Designer (v4.3.1). Synthesis of oligonucleotide probe sequences occurred on individual electrodes present on the semiconductor surface of the microarray by phosphoramidite chemistry in the $3^{\prime}$ to $5^{\prime}$ direction using standard software oligonucleotide pool synthesis settings and reagents prepared according to the manufacturer's protocols. Each sequence was synthesized in triplicate. After the synthesis of the unmodified oligonucleotide, $5^{\prime}$-biotin was added using a double coupling cycle with an extended 15 min coupling time. The final detritylation step was performed manually using the software by incubating the slides with TCA deblock ( $4 \times 30 \mathrm{~s}$ incubations) before washing the slide with acetonitrile four times and drying under argon. Oligonucleotides were then cleaved and deprotected on a stripping clamp system provided by the manufacturer using concentrated aqueous ammonia at $65^{\circ} \mathrm{C}$ for $18 \mathrm{~h}$. After cooling, the solution was recovered and the ammonia was removed by vacuum concentration. The oligonucleotide pool was purified using $2 \times$ illustra NAP-5 Columns (GE Life Sciences, PN: 17085302). The resulting solution was evaporated to dryness, resuspended in water and quantified by Nanodrop absorbance at $260 \mathrm{~nm}$. We used $\sim 0.1 \mathrm{fmol}$ of each individual oligonucleotide per enrichment reaction.

We ordered panels of double-stranded capture oligonucleotides from Twist Bioscience (Custom probes for NGS target enrichment). As recommended by Twist, we used $13.67 \mathrm{fmol}$ of each individual oligonucleotide per enrichment reaction.

Tiled-C-experimental procedure. For samples containing 100,000 cells or fewer, we followed a low-input $3 \mathrm{C}$ library preparation protocol ${ }^{50}$. Cells were sorted into 1 $\mathrm{ml}$ medium and fixed with $2 \%$ formaldehyde for $10 \mathrm{~min}$. After the reaction was quenched with glycine, cells were pelleted and washed with cold PBS. Following centrifugation, $\sim 5 \%$ supernatant was left behind to avoid disturbing the pellet. Cells were resuspended in cold lysis buffer $(10 \mathrm{mM}$ Tris $\mathrm{pH} 8,10 \mathrm{mM} \mathrm{NaCl}, 0.2 \%$ Igepal CA-630, 1x cOmplete Protease Inhibitor Cocktail, water) and snap frozen. Prior to digestion, cells were pelleted and all lysis buffer was removed by careful pipetting Chromatin was subsequently digested with the DpnII restriction enzyme in a $200 \mu \mathrm{l}$ reaction, to which three doses of $150 \mathrm{U}$ DpnII enzyme were added several hours apart and which was incubated $16-24 \mathrm{~h}$ at $37^{\circ} \mathrm{C}$. After heat-inactivation of DpnII, the ligation reaction was performed in the same tube, using $120 \mathrm{U}$ T4 ligase in an overnight incubation at $16^{\circ} \mathrm{C}$. Ligated DNA was reverse crosslinked and treated with proteinase $\mathrm{K}$ at $65^{\circ} \mathrm{C}$ in an overnight incubation. After RNase treatment, DNA was extracted using phenol-chloroform and transferred to a light phase lock tube for separation. To maximize yield, DNA was precipitated overnight in $70 \%$ ethanol at $-20^{\circ} \mathrm{C}$, after which DNA was pelleted and resuspended in PCR-grade water. The resulting $3 \mathrm{C}$ libraries were sonicated to $200 \mathrm{bp}$ fragments using a Covaris S220 Focused Ultrasonicator (six cycles of $60 \mathrm{~s}$; duty cycle: 10\%; intensity: 5; cycles per burst: 200). Illumina TruSeq adapters were subsequently added using the NEBNext Ultra II DNA Library Prep kit, according to the manufacturer's protocol. DNA clean-up steps were performed with Ampure XP beads at a 1:1.8 ratio to minimize loss of material. The libraries were indexed and amplified in 8-12 rounds of PCR amplification using the Herculase II PCR kit. $700 \mathrm{ng}-1 \mu \mathrm{g}$ of indexed material was used during subsequent capture-based enrichment.

When cell numbers were not limiting, we used aliquots of $\sim 10^{7}$ cells to prepare $3 \mathrm{C}$ libraries, following the efficient Capture-C protocol ${ }^{22}$. Cells were fixed with $2 \%$ formaldehyde for $10 \mathrm{~min}$, after which the reaction was quenched with glycine. The fixed cells were washed in cold PBS and resuspended in cold lysis buffer. After incubation on ice for $20 \mathrm{~min}$, the cells were snap frozen. Prior to digestion, the cells were resuspended in restriction buffer, Dounce homogenized on ice, and treated with SDS and Triton X-100. The chromatin was digested with DpnII, using three aliquots of $1500 \mathrm{U}$ DpnII restriction enzyme, which were added several hours apart over a total incubation time of $16-24 \mathrm{~h}$ at $37^{\circ} \mathrm{C}$. The digestion reaction was heatinactivated and digested chromatin was ligated overnight with $720 \mathrm{U}$ of T4 DNA ligase at $16^{\circ} \mathrm{C}$. The ligated DNA was reverse crosslinked and treated with proteinase $\mathrm{K}$ overnight at $65^{\circ} \mathrm{C}$. After RNase treatment, DNA was purified using phenol-chloroform extraction and precipitation with ethanol and sodium acetate at $-80^{\circ} \mathrm{C}$. The resulting $3 \mathrm{C}$ libraries were resuspended in PCR-grade water. Aliquots of 5-6 $\mu \mathrm{g}$ of 3C library were sonicated to $\sim 200 \mathrm{bp}$ fragments using a Covaris S220 Focused Ultrasonicator (six cycles of $60 \mathrm{~s}$; duty cycle: $10 \%$; intensity: 5 ; cycles per burst: 200). Illumina TruSeq adaptors were added using NEBNext DNA Library Prep reagents according to the manufacturer's protocol. The libraries were indexed and amplified using Agilent Herculase II reagents in a 6-cycle PCR reaction. DNA clean-up steps were performed using AMPure XP beads in a 1.8:1 bead:sample ratio. Where possible, we processed two aliquots of each sample in parallel and ligated the DNA with the same index to generate maximum library complexity. $1-1.5 \mu \mathrm{g}$ of indexed material was used during subsequent capture-based enrichment.

For enrichment using single-stranded oligonucleotides, we used the Nimblegen SeqCap EZ reagents and followed the SeqCap EZ Library SR User's Guide (Chapters 5-7). We multiplexed up to six samples per enrichment reaction in a single tube, and multiplied the volumes described in the protocol by the number of multiplexed libraries. Briefly, $700 \mathrm{ng}-1.5 \mu \mathrm{g}$ of indexed library (in exact 1:1 ratio if multiplexed reaction is performed) was mixed with $5 \mu \mathrm{g}$ mouse Cot-1 DNA, $1 \mathrm{nmol}$ of Nimblegen HE universal blocking oligonucleotides, and $1 \mathrm{nmol}$ of Nimblegen HE index-specific blocking oligonucleotides (corresponding to the Illumina TS index used) in a $1.5 \mu \mathrm{l}$ microcentrifuge tube. This mixture was dried completely in a vacuum centrifuge at $50^{\circ} \mathrm{C}$. The dried DNA was resuspended in $7.5 \mu \mathrm{l}$ Nimblegen 
hybridization buffer and $3 \mu \mathrm{l}$ Nimblegen hybridization component A and denatured at $95^{\circ} \mathrm{C}$ for $10 \mathrm{~min}$. Concurrently, $4.5 \mu \mathrm{l}$ of the biotinylated capture oligonucleotides was placed in a $200 \mu \mathrm{l}$ PCR tube and heated to $47^{\circ} \mathrm{C}$ in a PCR thermocycler. After denaturation, the $3 \mathrm{C}$ library mixture was added to the biotinylated oligonucleotides without removing them from the heating block in the thermocycler. The hybridization reaction was incubated in the thermocycler at $47^{\circ} \mathrm{C}$ for $64-72 \mathrm{~h}$ with a heated lid at $57^{\circ} \mathrm{C}$. After incubation was complete, we enriched for the captured DNA fragments using M270 streptavidin beads and the Nimblegen SeqCap EZ wash buffers, following the procedure described in the manufacturer's protocol. After the washing steps, the beads with captured material were resuspended in $40 \mu \mathrm{l}$ PCR-grade water. Captured DNA was amplified directly of the beads using the KAPA mastermix provided in the SeqCap EZ accessory kit $\mathrm{v} 2$, in two separate reactions of $\sim 10$ cycles, as described in the protocol. Ampure$\mathrm{XP}$ beads were used in a 1.8:1 bead:sample ratio to clean-up the amplification reaction and DNA was eluted in $30 \mu \mathrm{l} \mathrm{PCR-grade} \mathrm{water.} \mathrm{To} \mathrm{increase} \mathrm{enrichment,} \mathrm{a}$ second round of oligonucleotide capture was performed following the same procedure, using up to 2 ug of enriched material in a single hybridization reaction (even if multiplexed in first round) of 20-24 h.

For enrichment using double-stranded oligonucleotides, we used Twist Biosciences reagents and followed the Twist Custom Panel Protocol (Steps 4-7). To multiplex samples, we used $375-500 \mathrm{ng}$ indexed library per sample (in exact 1:1 ratio), mixed up to $1.5 \mu \mathrm{g}$ in a single tube, and used single reaction volumes as described in the protocol. We processed multiple tubes simultaneously if required. Streptavidin $\mathrm{C} 1$ beads were used to enrich the hybridized DNA and the washed material was amplified using 10-12 cycles of PCR. Ampure-XP beads were used in a 1.8:1 bead:sample ratio to clean-up the amplification reaction and DNA was eluted in $30 \mu \mathrm{l} \mathrm{PCR}$-grade water. To increase enrichment, a second round of oligonucleotide capture was performed following the same procedure, using up to $1.5 \mu \mathrm{g}$ of enriched material in a single hybridization reaction (even if multiplexed in first round) of $20-24 \mathrm{~h}$.

All Tiled-C data in this study were generated using a double capture enrichment procedure, since we have previously shown that this substantially increases enrichment in the Capture-C approach ${ }^{22}$. However, we have recently noticed that a single Tiled-C capture step results in very efficient enrichment ( $\sim 85 \%$ capture efficiency), which is only marginally improved after a second capture step $(\sim 88 \%$ capture efficiency). It might therefore be preferable to perform a single enrichment step as this is less labor-intensive, cheaper and results in fewer PCR duplicates.

The enriched Tiled-C libraries were assessed using the Agilent Bioanalyzer or D1000 Tapestation and quantified using KAPA Library Quantification reagents, before sequencing using the Illumina NextSeq platform. In high-quality libraries, sequencing 3-5 million reads per enriched $\mathrm{Mb}$ per sample is sufficient for data at 5 $\mathrm{kb}$ resolution.

Tiled-C-analysis. The most straightforward way to analyze Tiled-C data is to use the HiCPro pipeline ${ }^{51}$ with the options for Capture-Hi-C analysis. We have also adjusted our pipelines for Capture-C analysis to be compatible with Tiled-C data. This pipeline is designed to analyze deep, targeted $3 \mathrm{C}$ data and provides very stringent filtering of $3 \mathrm{C}$ artefacts, including PCR duplicates. All data presented in the paper have been analyzed using a combination of this CCseqBasic pipeline (https://github.com/Hughes-Genome-Group/CCseqBasicF/releases), custom scripts and ICE normalization ${ }^{52}$. Detailed instructions and custom scripts are available on https://github.com/oudelaar/TiledC. Interaction profiles from virtual viewpoints (Supplementary Fig. 1) can be generated using the CCseqBasic pipeline with the options for Capture-C analysis.

When Tiled-C data are compared between different cell types (Figs. 1b, c, 4, 5; Supplementary Figs. 2, 7-12), we have down-sampled the data in the different samples to an equal number of valid interactions within the tiled region.

Quantification of enhancer-promoter interactions of interest was performed based on interaction counts in the corresponding bins after normalizing for the total number of counts in the matrix. We used the following coordinates for quantification of regulatory elements of interest (highlighted in Supplementary Figs. 10-12):

a-globin: chr11:32,145,000-32,148,000; chr11:32,182,000-32,185,000; chr11:32,195,000-32,198,000;

Slc25a37: chr14:69,902,000-69,905,000; chr14:69,939,000-69,942,000; Tall: chr4:114,728,000-114,731,000; chr4:114,768,000-114,771,000;

Cd47: chr16:49,770,000-49,773,000 and chr16:49,852,000-49,855,000

Cpeb4: chr11:31,711,000-31,714,000; chr11:31,770,000-31,773,000;

Btg: chr1:135,995,000-135,998,000; chr1:135,975,000-135,978,000.

We identified TADs based on insulation indices using TADtool ${ }^{53}$.

To examine the reproducibility of Tiled-C in low-input samples, we used HiCRep $^{54}$ to calculate stratum-adjusted correlation coefficients, considering a maximum distance of 100,000 bp.

Hi-C. We compared Tiled-C data in mouse ES cells to the deepest currently available Hi-C data in mouse ES cells ${ }^{9}$. We explored the data using HiGlass ${ }^{55}$ and downloaded and reanalyzed the Hi-C data using the HiC-Pro pipeline ${ }^{51}$ with default options and ICE normalization ${ }^{52}$.
ATAC-seq-experimental procedure. For FACS-sorted erythroid progenitors from fetal liver, either two or three replicates were processed of $\sim 50,000$ cells each for each sorted population. ATAC-seq was performed as previously described ${ }^{36}$.

For FACS-sorted hematopoietic stem and progenitor cells from adult bone marrow, one replicate of between 5000 and 20,000 cells was processed for each population. Cells were spun at $500 \times g$ for $10 \mathrm{~min}$ at $4{ }^{\circ} \mathrm{C}$. The supernatant was discarded and cells were resuspended directly in Dig-transposition buffer $(25 \mu \mathrm{l} 2 \mathrm{x}$ TD Buffer [Illumina], $2.5 \mu \mathrm{l}$ Tn5 transposase, $0.5 \mu \mathrm{l}$ \% digitonin and $22 \mu \mathrm{l} \mathrm{H}_{2} \mathrm{O}$ ) before incubating at $37^{\circ} \mathrm{C}$ for $30 \mathrm{~min}$ with agitation at $600 \mathrm{rpm}$. After the transposition step, samples were processed as previously described ${ }^{36}$.

ATAC-seq-analysis. Reads were mapped to the mouse $\mathrm{mm} 9$ genome and PCR duplicates removed using NGseqBasic ${ }^{56}$. Technical replicates were merged and peaks called using MACS2 ${ }^{57}$. Peaks were merged and the number of reads in each sample overlapping each peak was calculated using BEDTools merge and multicov ${ }^{58}$. For visualization, bedgraph files were generated using BEDTools genomecov with a scaling factor of 1e6/(total number of reads in peaks). All analysis scripts are available at https://github.com/rbeagrie/alpha-tiledc.

Single-cell RNA-seq-experimental procedure. Fetal livers were harvested and pooled from 7 e13.5 C57BL/6 mouse embryos and processed as above. Cells were first stained with $2.5 \mu \mathrm{g} / \mathrm{ml}$ biotin-conjugated anti-Ter119 (BD 553672) and $2.5 \mu \mathrm{g} /$ $\mathrm{ml}$ purified anti-Ter119 (BioLegend 116241) conjugated to a DNA oligonucleotide (ADT2 GAGGCGATTGAT). After magnetic depletion, an equal number of Ter119+ cells were added back to the Ter119- fraction for further analysis, ensuring a balanced distribution of erythroid cells from all stages of differentiation. Cells were then stained with a panel of 9 further DNA-oligonucleotide-conjugated antibodies $(0.33 \mu \mathrm{g} / \mathrm{ml}$ anti-CD71: BioLegend 113802 conjugated with ADT9 [CGAAGAAGGAGT], $1 \mu \mathrm{g} / \mathrm{ml}$ anti-CD41: BioLegend 133919 conjugated with ADT3 [TGTCCGGCAATA], $1 \mu \mathrm{g} / \mathrm{ml}$ anti-CD45R: BioLegend 103249 conjugated with ADT5 [GATCGTAATACC], $1 \mu \mathrm{g} / \mathrm{ml}$ anti-CD3e: BioLegend $100345 \mathrm{con}-$ jugated with ADT7 [CATCGGTGTACA], $1 \mu \mathrm{g} / \mathrm{ml}$ anti-CD11b: BioLegend 101249 conjugated with ADT1 [CATGATTGGCTC], $1 \mu \mathrm{g} / \mathrm{ml}$ anti-Ly6G/6C: BioLegend 108449 conjugated with ADT4 [TGGTGAACCTGG], $1 \mu \mathrm{g} / \mathrm{ml}$ anti-CD44: BioLegend 103051 conjugated with ADT8 [GTCTAGACTTCG], $1 \mu \mathrm{g} / \mathrm{ml}$ anti-cKit: BioLegend 105829 conjugated with ADT6 [AAGCGCTTGGCA], and $1 \mu \mathrm{g} / \mathrm{ml}$ nonspecific IgG conjugated with ADT10 [CGGAGTAGTAAT]). Antibodies were conjugated to streptavidin as previously described ${ }^{35}$ and mixed with biotinylated custom oligonucleotides. Cells were processed for single-cell RNA-seq using the 10x Genomics Single Cell 3' v2 kit.

Single-cell RNA-seq-analysis. cDNA reads were mapped to the mouse mm10 assembly and barcodes assigned to cells using Cell Ranger v2.1.1 (10x Genomics) ADT reads were mapped to cell/antibody barcodes using CITE-seq-count (https:// github.com/Hoohm/CITE-seq-Count). Potential doublet cells were removed using Scrublet ${ }^{59}$. Further analysis was performed using Seurat v2. Low quality cells with less than 300 or more than 5000 identified genes, or with more than $9 \%$ mitochondrial reads were also removed. Clusters were identified using the "FindClusters" function and UMAP projection was generated using the "RunUMAP" function, both with the first 16 principle components. Seurat clusters were annotated using marker genes and by reference to previously published data ${ }^{34}$-Seurat identified two clusters corresponding to committed erythroid progenitors (CEP) and four clusters corresponding to cells undergoing erythroid terminal differentiation (ETD). Average gene expression for populations matching those obtained by FACS sorting was generated by using the "SubsetData" function to select cells with low levels of cell-surface barcodes corresponding to lineage markers, and appropriate levels of barcodes corresponding to CD71 and Ter119. All analysis scripts are available at https://github.com/rbeagrie/alpha-tiledc.

RNA-FISH-experimental procedure. Standard RNA-FISH was carried out as previously described ${ }^{60}$. Sorted cells from mouse fetal liver were placed back into culture for $6 \mathrm{~h}$ to allow nascent transcription to be re-established, washed twice with PBS and spotted onto poly-l-lysine coated slides before being immersed in $3.7 \%$ formaldehyde in PBS for $20 \mathrm{~m}$ at RT. Slides were then washed $3 \mathrm{x}$ with PBS and stored in $70 \%$ ethanol at $-20^{\circ} \mathrm{C}$. Slides were then rinsed in TS $[0.15 \mathrm{M} \mathrm{NaCl}$ and $0.1 \mathrm{M}$ Tris- $\mathrm{HCl}]$, incubated with $0.02 \%$ pepsin in $0.01 \mathrm{M} \mathrm{HCl}$ at $37^{\circ} \mathrm{C}$ for 5 min, rinsed with water and post-fixed in $3.7 \%$ formaldehyde for $5 \mathrm{~m}$ at RT. Slides were then washed and dehydrated with successive PBS, $70 \%$ ethanol, $90 \%$ ethanol, and $100 \%$ ethanol before air drying. Samples were hybridized overnight at $37^{\circ} \mathrm{C}$ with digoxygenin-labelled oligonucleotide probes directed to a-globin introns (pool of four oligos: mouse-alpha-1 [agctgcccactgagcgagtgccaggtccat], mouse-alpha-2 [gagaagttgaggtcacagaaaagcatagtt], mouse-alpha-3 [cttcctaggggtcccagatgccgcctgcca] and mouse-alpha-4 [ccactatgttccctgccttgggcacgagga]; $30 \mathrm{ng}$ per slide in hybridization mastermix [ $25 \%$ formamide, $200 \mathrm{ng} / \mathrm{mL}$ salmon sperm DNA, $5 \times$ Denhardt's solution, $50-\mathrm{mM} \mathrm{NaPO}_{4}, 1 \mathrm{mM}$ EDTA in $2 \times \mathrm{SSC}$ ]). Slides were then washed $4 \times$ with $2 \times$ SSC [ $300 \mathrm{mM} \mathrm{NaCl}$ and $30 \mathrm{mM}$ Trisodium citrate] and $1 \times$ with TST [0.15 $\mathrm{M} \mathrm{NaCl}, 0.1 \mathrm{M}$ Tris- $\mathrm{HCl}$ and $0.05 \%(\mathrm{v} / \mathrm{v})$ Tween 20 ] before incubation for $30 \mathrm{~m}$ at RT with $100 \mu \mathrm{l}$ blocking solution [1.35\% ELISA blocking reagent (Roche)]. For visualization, slides were washed $1 \times$ with TST, incubated with $100 \mu \mathrm{l}$ sheep anti- 
DIG FITC [Roche 11207741910] 1:50 in blocking solution for $30 \mathrm{~m}$ at RT, washed $2 \times$ with TST, incubated with $100 \mu \mathrm{l}$ rabbit anti-sheep FITC [Vector FI-6000] 1:100 in blocking solution for $30 \mathrm{~m}$ at RT, washed $1 \times$ with PBS and post-fixed in $3.7 \%$ formaldehyde for $5 \mathrm{~m}$ at RT. Slides were then washed and dehydrated with successive PBS, 70\% ethanol, 90\% ethanol, and 100\% ethanol before air drying. Finally, coverslips were mounted with $10 \mu \mathrm{l}$ mounting media $[1 \mathrm{mg} / \mathrm{mL}$ DAPI in Vectashield antifade mounting medium (Vector)]. Two negative controls were also included: brain tissue from a male, adult CD1 mouse and Ter119+ (i.e., mature) fetal liver erythroid cells that were probed with secondary antibody but no primary antibody. Magnetically purified but not FACS-purified Ter119+ fetal liver erythroid cells were used as a positive control.

RNA-FISH-imaging equipment and settings. Widefield fluorescence imaging was performed at $20^{\circ} \mathrm{C}$ on a DeltaVision Elite system (Applied Precision) equipped with a $\times 100 / 1.40$ NA UPLSAPO oil immersion objective (Olympus), a CoolSnap HQ2 CCD camera (Photometrics), DAPI (excitation 390/18; emission 435/40) and FITC (excitation 475/28; emission 525/45) filters. 12-bit image stacks were acquired with a $\mathrm{z}$-step of $150 \mathrm{~nm}$ giving a voxel size of $64.5 \times 64.5 \times 150 \mathrm{~nm}$.

RNA-FISH-image analysis. Image analysis was blinded by renaming image files from all experiments with random character strings and processing them together. Images were manually examined, and each cell was scored for the presence of active nascent-transcription foci. Analysis scripts are available at https://github. com/rbeagrie/alpha-tiledc.

Reporting summary. Further information on research design is available in the Nature Research Reporting Summary linked to this article.

\section{Data availability}

The data that support this study are available from the corresponding author upon reasonable request. Tiled-C, ATAC-seq and single-cell RNA-seq data generated in this study have been deposited in the NCBI Gene Expression Omnibus under accession code GSE137477. All RNA-FISH image files are archived in the Imaging Data Resource under accession code idr0084. A UCSC hub for visualizing ATAC-seq and single-cell RNA-seq mean expression data is available at http://sara.molbiol.ox.ac.uk/public/hugheslab/alphatiledc/hub.txt. The source data underlying Fig. 3e are provided as a Source Data file.

\section{Code availability}

Tiled-C data were analyzed with the CCseqBasic pipeline and custom scripts available at https://github.com/Hughes-Genome-Group/CCseqBasicF/releases and https://github. com/oudelaar/TiledC. ATAC-seq data were analyzed with the NGseqBasic pipeline ${ }^{56}$ and scripts available at https://github.com/rbeagrie/alpha-tiledc. Single-cell RNA-seq data were analyzed with scripts available at https://github.com/Hoohm/CITE-seq-Count and https://github.com/rbeagrie/alpha-tiledc. RNA-FISH analysis was performed using scripts available at https://github.com/rbeagrie/alpha-tiledc.

Received: 6 February 2020; Accepted: 13 May 2020;

Published online: 01 June 2020

\section{References}

1. Furlong, E. E. M. \& Levine, M. Developmental enhancers and chromosome topology. Science 361, 1341-1345 (2018).

2. Schoenfelder, S. \& Fraser, P. Long-range enhancer-promoter contacts in gene expression control. Nat. Rev. Genet. 20, 437-455 (2019).

3. Stadhouders, R., Filion, G. J. \& Graf, T. Transcription factors and 3D genome conformation in cell-fate decisions. Nature 569, 345-354 (2019).

4. Andrey, G. \& Mundlos, S. The three-dimensional genome: regulating gene expression during pluripotency and development. Development 144, 3646-3658 (2017).

5. de Laat, W. \& Duboule, D. Topology of mammalian developmental enhancers and their regulatory landscapes. Nature 502, 499-506 (2013).

6. Zheng, H. \& Xie, W. The role of 3D genome organization in development and cell differentiation. Nat. Rev. Mol. Cell Biol. 34, 903-916 (2019).

7. Phillips-Cremins, J. E. et al. Architectural protein subclasses shape 3D organization of genomes during lineage commitment. Cell 153, 1281-1295 (2013).

8. Dixon, J. R. et al. Chromatin architecture reorganization during stem cell differentiation. Nature 518, 331-336 (2015).

9. Bonev, B. et al. Multiscale 3D genome rewiring during mouse neural development. Cell 171, 557-572.e524 (2017).

10. Stadhouders, R. et al. Transcription factors orchestrate dynamic interplay between genome topology and gene regulation during cell reprogramming. Nat. Genet. 50, 238-249 (2018).
11. Montavon, T. et al. A regulatory archipelago controls Hox genes transcription in digits. Cell 147, 1132-1145 (2011).

12. Williamson, I., Lettice, L. A., Hill, R. E. \& Bickmore, W. A. Shh and ZRS enhancer colocalisation is specific to the zone of polarising activity. Development 143, 2994-3001 (2016).

13. Andrey, G. et al. Characterization of hundreds of regulatory landscapes in developing limbs reveals two regimes of chromatin folding. Genome Res. 27, 223-233 (2017)

14. Freire-Pritchett, P. et al. Global reorganisation of cis-regulatory units upon lineage commitment of human embryonic stem cells. eLife 6, e21926 (2017).

15. Rubin, A. J. et al. Lineage-specific dynamic and pre-established enhancer-promoter contacts cooperate in terminal differentiation. Nat. Genet. 49, 1522-1528 (2017).

16. Jin, F. et al. A high-resolution map of the three-dimensional chromatin interactome in human cells. Nature 503, 290-294 (2013).

17. Ghavi-Helm, Y. et al. Enhancer loops appear stable during development and are associated with paused polymerase. Nature 513, 89-100 (2014).

18. Paliou, C. et al. Preformed chromatin topology assists transcriptional robustness of Shh during limb development. Proc. Natl. Acad. Sci. USA 116, 12390-12399 (2019)

19. Dekker, J., Rippe, K., Dekker, M. \& Kleckner, N. Capturing chromosome conformation. Science 295, 1306-1311 (2002).

20. Oudelaar, A. M., Davies, J. O. J., Downes, D. J., Higgs, D. R. \& Hughes, J. R. Robust detection of chromosomal interactions from small numbers of cells using low-input Capture-C. Nucleic Acids Res. https://doi.org/10.1093/nar/ gkx1194 (2017).

21. Hughes, J. R. et al. Analysis of hundreds of cis-regulatory landscapes at high resolution in a single, high-throughput experiment. Nat. Genet. 46, 205-212 (2014).

22. Davies, J. O. J. et al. Multiplexed analysis of chromosome conformation at vastly improved sensitivity. Nat. Methods 86, 1202-1210 (2015).

23. Lieberman-Aiden, E. et al. Comprehensive mapping of long-range interactions reveals folding principles of the human genome. Science 326, 289-293 (2009).

24. Simonis, M. et al. Nuclear organization of active and inactive chromatin domains uncovered by chromosome conformation capture-on-chip (4C). Nat. Genet. 38, 1348-1354 (2006).

25. Zhao, Z. et al. Circular chromosome conformation capture (4C) uncovers extensive networks of epigenetically regulated intra- and interchromosomal interactions. Nat. Genet. 38, 1341-1347 (2006).

26. Kolovos, P. et al. Targeted Chromatin Capture (T2C): a novel high resolution high throughput method to detect genomic interactions and regulatory elements. Epigenetics Chromatin 7, 10 (2014).

27. Dryden, N. H. et al. Unbiased analysis of potential targets of breast cancer susceptibility loci by Capture Hi-C. Genome Res. 24, 1854-1868 (2014).

28. Sanborn, A. L. et al. Chromatin extrusion explains key features of loop and domain formation in wild-type and engineered genomes. Proc. Natl. Acad. Sci. USA 112, E6456-6465 (2015).

29. Hay, D. et al. Genetic dissection of the $\alpha$-globin super-enhancer in vivo. Nat. Genet. 48, 895-903 (2016).

30. Oudelaar, A. M. et al. Single-allele chromatin interactions identify regulatory hubs in dynamic compartmentalized domains. Nat. Genet. 50, 1744-1751 (2018).

31. Hanssen, L. L. P. et al. Tissue-specific CTCF-cohesin-mediated chromatin architecture delimits enhancer interactions and function in vivo. Nat. Cell Biol. 19, 952-961 (2017).

32. Chiariello, A. M. et al. A dynamic folded hairpin conformation is associated with $\alpha$-globin activation in erythroid cells. Cell Rep. 30, 2125-2135.e2125 (2020).

33. Pop, R. et al. A key commitment step in erythropoiesis is synchronized with the cell cycle clock through mutual inhibition between PU.1 and S-phase progression. PLoS Biol. 8, e1000484 (2010).

34. Tusi, B. K. et al. Population snapshots predict early haematopoietic and erythroid hierarchies. Nature 555, 54-60 (2018).

35. Stoeckius, M. et al. Simultaneous epitope and transcriptome measurement in single cells. Nat. Methods 14, 865-868 (2017).

36. Buenrostro, J. D., Giresi, P. G., Zaba, L. C., Chang, H. Y. \& Greenleaf, W. J. Transposition of native chromatin for fast and sensitive epigenomic profiling of open chromatin, DNA-binding proteins and nucleosome position. Nat. Methods 10, 1213-1218 (2013).

37. Oudelaar, A. M. et al. A revised model for promoter competition based on multi-way chromatin interactions at the $\alpha$-globin locus. Nat. Commun. 10, 5412-5418 (2019)

38. Oudelaar, A. M. et al. Between form and function: the complexity of genome folding. Hum. Mol. Genet. 26, R208-R215 (2017).

39. Anguita, E. et al. Globin gene activation during haemopoiesis is driven by protein complexes nucleated by GATA-1 and GATA-2. EMBO J. 23, 2841-2852 (2004).

40. Palstra, R.-J. et al. The beta-globin nuclear compartment in development and erythroid differentiation. Nat. Genet. 35, 190-194 (2003). 
41. Vernimmen, D., De Gobbi, M., Sloane-Stanley, J. A., Wood, W. G. \& Higgs, D. R. Long-range chromosomal interactions regulate the timing of the transition between poised and active gene expression. EMBO J. 26, 2041-2051 (2007).

42. Brown, J. M. et al. A tissue-specific self-interacting chromatin domain forms independently of enhancer-promoter interactions. Nat. Commun. 9, 376 (2018).

43. Allahyar, A. et al. Enhancer hubs and loop collisions identified from singleallele topologies. Nat. Genet. 295, 1306 (2018).

44. Chen, H. et al. Dynamic interplay between enhancer-promoter topology and gene activity. Nat. Genet. 50, 1296-1303 (2018).

45. Fudenberg, G., Abdennur, N., Imakaev, M., Goloborodko, A. \& Mirny, L. A. Emerging evidence of chromosome folding by loop extrusion. Cold Spring Harb. Symp. Quant. Biol. https://doi.org/10.1101/sqb.2017.82.034710 (2018).

46. Kagey, M. H. et al. Mediator and cohesin connect gene expression and chromatin architecture. Nature 467, 430-435 (2010).

47. Schmidt, D. et al. A CTCF-independent role for cohesin in tissue-specific transcription. Genome Res. 20, 578-588 (2010).

48. Pietras, E. M. et al. Functionally distinct subsets of lineage-biased multipotent progenitors control blood production in normal and regenerative conditions. Cell Stem Cell 17, 35-46 (2015).

49. Dostie, J. et al. Chromosome Conformation Capture Carbon Copy (5C): a massively parallel solution for mapping interactions between genomic elements. Genome Res. 16, 1299-1309 (2006).

50. Oudelaar, A. M., Downes, D., Davies, J. \& Hughes, J. Low-input Capture-C: a chromosome conformation capture assay to analyze chromatin architecture in small numbers of cells. Bio-protocol https://doi.org/10.21769/BioProtoc.2645 (2017).

51. Servant, N. et al. HiC-Pro: an optimized and flexible pipeline for $\mathrm{Hi}-\mathrm{C}$ data processing. Genome Biol. 16, 259 (2015).

52. Imakaev, M. et al. Iterative correction of Hi-C data reveals hallmarks of chromosome organization. Nat. Methods 9, 999-1003 (2012).

53. Kruse, K., Hug, C. B., Hernández-Rodríguez, B. \& Vaquerizas, J. M. TADtool: visual parameter identification for TAD-calling algorithms. Bioinformatics 32, 3190-3192 (2016).

54. Yang, T. et al. HiCRep: assessing the reproducibility of Hi-C data using a stratum-adjusted correlation coefficient. Genome Res. 27, 1939-1949 (2017)

55. Kerpedjiev, P. et al. HiGlass: web-based visual exploration and analysis of genome interaction maps. Genome Biol. 19, 125-112 (2018).

56. Telenius, J. \& Hughes, J. R. NGseqBasic-a single-command UNIX tool for ATAC-seq, DNaseI-seq, Cut-and-Run, and ChIP-seq data mapping, highresolution visualisation, and quality control. bioRxiv, https://doi.org/10.1101/ 393413 (2018)

57. Zhang, Y. et al. Model-based analysis of ChIP-Seq (MACS). Genome Biol. 9, R137 (2008).

58. Quinlan, A. R. \& Hall, I. M. BEDTools: a flexible suite of utilities for comparing genomic features. Bioinforma. (Oxf., Engl.) 26, 841-842 (2010).

59. Wolock, S. L., Lopez, R. \& Klein, A. M. Scrublet: computational identification of cell doublets in single-cell transcriptomic data. Cell Syst. 8, 281-291.e289 (2019).

60. Brown, J. M. \& Buckle, V. J. Detection of nascent RNA transcripts by fluorescence in situ hybridization. Methods Mol. Biol. 659, 33-50 (2010).

\section{Acknowledgements}

We thank J. Sloane-Stanley and J. Sharpe of the WIMM Transgenics Facility for mouse breeding and fetal liver provision, K. Clark, S.-A. Clark and C. Waugh of the WIMM
Flow Cytometry Facility for cell sorting services, N. Ashley of the WIMM Single Cell facility for antibody conjugation and 10X library preparation services, J. Brown for help with RNA-FISH, D. Downes for help with ATAC-seq and C. Garnett for advice on lowinput ATAC-seq. We wish to acknowledge the Centre for Computational Biology, MRC Weatherall Institute of Molecular Medicine, Radcliffe Department of Medicine, and University of Oxford for use of their services in this project. This work was supported by the Medical Research Council (MC_UU_00016 to D.R.H., J.R.H., and V.J.B. and MR/ N00969X/1 to J.R.H., T.B., and V.J.B.), Wellcome Trust (209181/Z/17/Z to R.A.B.), National Institutes of Health (NIH NIDDK R01DK100915 to M.S.), Biotechnology and Biological Sciences Research Council (BB/J001694/2 and BB/ R008655/1 to A.H.E.-S. and BB/M025624/1 to A.S.) and a Stevenson Junior Research Fellowship at University College, Oxford (to A.M.O.).

\section{Author contributions}

A.M.O. and R.A.B. performed experiments and bioinformatic analyses and wrote the manuscript. M.G., E.G., D.H., and J.C. performed experiments. S.d.O., A.S., A.H.E.-S., and T.B. synthesized oligonucleotides. J.K. wrote the oligonucleotide design tool. J.M.T. performed bioinformatic analyses. V.J.B. and M.S. provided conceptual advice. D.R.H. and J.R.H. supervised the project and wrote the manuscript.

\section{Competing interests}

J.R.H. is founder and shareholder of Nucleome Therapeutics.

\section{Additional information}

Supplementary information is available for this paper at https://doi.org/10.1038/s41467020-16598-7.

Correspondence and requests for materials should be addressed to D.R.H. or J.R.H.

Peer review information Nature Communications thanks the anonymous reviewer(s) for their contribution to the peer review of this work. Peer reviewer reports are available.

Reprints and permission information is available at http://www.nature.com/reprints

Publisher's note Springer Nature remains neutral with regard to jurisdictional claims in published maps and institutional affiliations.

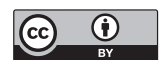

Open Access This article is licensed under a Creative Commons Attribution 4.0 International License, which permits use, sharing, adaptation, distribution and reproduction in any medium or format, as long as you give appropriate credit to the original author(s) and the source, provide a link to the Creative Commons license, and indicate if changes were made. The images or other third party material in this article are included in the article's Creative Commons license, unless indicated otherwise in a credit line to the material. If material is not included in the article's Creative Commons license and your intended use is not permitted by statutory regulation or exceeds the permitted use, you will need to obtain permission directly from the copyright holder. To view a copy of this license, visit http://creativecommons.org/ licenses/by/4.0/.

(c) The Author(s) 2020 